\section{TATRA \\ MOUNTaiNS \\ Mathematical Publications}

DOI: $10.2478 / \mathrm{tmmp}-2014-0032$

Tatra Mt. Math. Publ. 61 (2014), 131-140

\title{
MOMENTS OF MARKOV-SWITCHING MODELS
}

\author{
Anna PetričKová
}

\begin{abstract}
In this paper we have focused on the class of regime-switching time series models with regimes determined by unobservable variables, concretely Markov-switching models. We have derived 2nd central moment of the MSW models for two cases - state-independent and state-dependent model.
\end{abstract}

\section{Introduction}

In the real life we often meet time series that exhibit strong non-linear features, because linear models are not in general always suitable for use. Markov-switching models (or MSW models), proposed by Hamilton [2] and belonging to this group of the models have been successfully used for fitting a lot of economic and financial time series, for example, interest rates, exchange rates, consumption costs and so on.

The goal of our work is to derive the second central moment, firstly for the state-independent and then for the state-dependent autoregressive MSW models. We have been inspired by Timmermann's paper [4] for the special form of the Markov-switching models.

The paper is organized as follows. After this introduction, the theoretical basis of the non-linear Markov-switching models is given, followed by the derivation of the second central moment (variance) of the state-independent and state-dependent MSW models. For both cases it also follows comparison of the calculation of the variance computed with classical formula, defined in the program Mathematica, version 8 , and the formula derived for the illustrative data series (concretely from hydrology). Finally we briefly summarize the results.

(C) 2014 Mathematical Institute, Slovak Academy of Sciences.

2010 Mathematics Subject Classification: 37M10.

Keywords: time series, non-linear models, Markov-switching model, central moment.

This work was supported by VEGA 1/0143/11. 


\section{ANNA PETRIČKOVÁ}

\section{Overview of Markov-switching (MSW) models}

In this work we focus on the class of non-linear regime-switching models assuming that the regime that occurs at time $t$ cannot be observed. It is determined by an unobservable process, which we denote as $S_{t}$. It follows that separate regimes cannot be identified exactly, but only with some probability. In case of 2 regimes process $\left\{S_{t}\right\}$ can take only values 1 and 2 . If we work with a model $\operatorname{AR}(p)$, where $p$ denotes the order of the MSW process, the regime-switching model with regimes determined by unobservable variables is

$$
X_{t}=\phi_{0, S_{t}}+\phi_{1, S_{t}} X_{t-1}+\cdots+\phi_{p, S_{t}} X_{t-p}+\varepsilon_{t}, \quad S_{t}=1,2 .
$$

We assume that the random variable $\varepsilon_{t}$ is identically, independently and normally distributed with zero mean, that is $\varepsilon_{t} \sim N\left(0, \sigma_{\varepsilon}^{2}\right)$.

The class of MSW models with $N$ regimes is based on the assumption that a regime is determined by the discrete ergodic first order Markov process, hence it is important to consider only the actual and the previous state

$$
P\left(q_{t}=S_{j} \mid q_{t-1}=S_{i}, q_{t-2}=S_{k}, \ldots\right)=P\left(q_{t}=S_{j} \mid q_{t-1}=S_{i}\right)=p_{i j}
$$

for $1 \leq i, j \leq N$, where $p_{i j}$ is a state transition probability (transition from the state $S_{i}$ at the time $t-1$ to the state $S_{j}$ at time $t$ in the Markov chain), $0 \leq p_{i j} \leq 1, \sum_{j=1}^{N} p_{i j}=1, t=1,2, \ldots, n$ are time instants associated with state changes and $q_{t}$ is the actual state at time $t$. For details on MSW models see, e.g., [1] 3 .

\section{Moments of Markov-switching models}

\subsection{The relation between conditional and unconditional central moments of MSW model}

The state-dependent $p$ th order autoregressive MSW model with normally distributed increments is described by

$$
X_{t}=\phi_{0, q_{t}}+\phi_{1, q_{t}} X_{t-1}+\cdots+\phi_{p, q_{t}} X_{t-p}+\varepsilon_{t},
$$

where $\left\{X_{t}\right\}, t=1, \ldots, T$ is a stochastic process generating an examined time series, $q_{t}=1,2, \ldots, m$ is a (unobservable) state variable, generated by an ergodic $m$-state Markov chain, $q_{t} \in M=\{1,2, \ldots, m\}$ and random variables $\varepsilon_{t}$ are IID and normally distributed with zero mean.

We assume that the number of states $N$ is finite and coefficients $\phi_{j}, j=1, \ldots, p$ are state dependent (coefficients of autoregressive model may be different 
in each regime), $\boldsymbol{\pi}=\left(\pi_{1}, \pi_{2}, \ldots, \pi_{N}\right)^{\prime}$ is the $N$-vector of steady-state (ergodic) probabilities that solve the system of equations $\mathbf{P}^{\prime} \boldsymbol{\pi}=\boldsymbol{\pi}$, where

$$
\mathbf{P}=\left(\begin{array}{ccc}
p_{11} & \cdots & p_{1 N} \\
\vdots & \ddots & \vdots \\
p_{N 1} & \cdots & p_{N N}
\end{array}\right)
$$

is the transition probability matrix of the type $N \times N$. The vector $\pi$ represents unconditional probabilities, applying to the $N$ states.

In general for $N$-states the $k$ th order unconditional central moment of $\left\{X_{t}\right\}$ is defined as

$$
\begin{aligned}
\mu^{k}=E\left[\left(X_{t}-\mu\right)^{k}\right] & =\sum_{i=1}^{N} E\left[\left(X_{t}-\mu\right)^{k} \mid q_{t}=i\right] \cdot P\left[q_{t}=i\right] \\
& =\sum_{i=1}^{N} E\left[\left(X_{t}-\mu\right)^{k} \mid q_{t}=i\right] \cdot \pi_{i} \\
& =\sum_{i=1}^{N} \mu_{i}^{k} \pi_{i}=\boldsymbol{\pi}^{\prime}\left(\begin{array}{c}
\mu_{1}^{k} \\
\vdots \\
\mu_{N}^{k}
\end{array}\right),
\end{aligned}
$$

where $\pi_{i}$ is the unconditional probability of state $i$ and $\mu_{i}^{k}, i=1, \ldots, N$ is the conditional $k$ th order central moment for the $i$ th regime of the stochastic process $\left\{X_{t}\right\}$.

The problem is that we do not know conditional central moments for each state (we only calculate conditional means). Therefore, we need derive formulas for the computation of unconditional central moments.

Let B be the $(N \times N)$ matrix of transition probabilities for the "time-reversed" Markov chain that moves back in time, and $b_{i, j}$ its element in the $i$ th row and the $j$ th column, so that

From

$$
P\left(q_{t}=S_{j} \mid q_{t+1}=S_{i}\right)=b_{i j}, \quad 0 \leq b_{i j} \leq 1, \quad \sum_{j=1}^{N} b_{i j}=1 .
$$

$$
\begin{aligned}
P\left(q_{t}=S_{j} \cap q_{t+1}=S_{i}\right) & =P\left(q_{t+1}=S_{i} \mid q_{t}=S_{j}\right) \cdot P\left(q_{t}=S_{j}\right) \\
& =P\left(q_{t}=S_{j} \mid q_{t+1}=S_{i}\right) \cdot P\left(q_{t+1}=S_{i}\right)
\end{aligned}
$$

the "backward" transition probability matrix B is related to the "forward" transition probabilities as follows

$$
b_{i j}=p_{j i}\left(\frac{\pi_{j}}{\pi_{i}}\right), \quad i, j=1, \ldots, N .
$$




\section{ANNA PETRIČKOVÁ}

\subsection{State-independent MSW models}

Before the derivation of the moment, firstly let us assume the simple autoregressive first order MSW model

$$
X_{t}=\phi_{q_{t}}+\phi_{1} X_{t-1}+\varepsilon_{t},
$$

thus $\phi_{1, q_{t}}=\phi_{1}$ and $\phi_{0, q_{t}}=\phi_{q_{t}}$. Consider 3-regime MSW model $\left(q_{t} \in\{1,2,3\}\right)$.

For the state-dependent mean value we have

$$
\mu_{q_{t}}=\phi_{q_{t}}+\phi_{1} \mu_{q_{t-1}} .
$$

When we substitute $\phi_{q_{t}}$ into the basic model we get

$$
X_{t}=\mu_{q_{t}}+\phi_{1}\left(X_{t-1}-\mu_{q_{t-1}}\right)+\varepsilon_{t},
$$

after substituting backwards and considering to the stationarity of the process $\left(\left|\phi_{1}\right|<1\right)$, we obtain

$$
X_{t}-\mu_{q_{t}}=\sum_{i=0}^{\infty} \phi_{1}^{i} \varepsilon_{t-i} .
$$

From the assumptions $E\left[\varepsilon_{t-i} \mid \mathbf{q}_{\mathbf{t}}\right]=\mathbf{0}, E\left[\varepsilon_{t-i}\right]=0$, it holds that the first moment $E\left[\left(X_{t-1}-\mu_{q_{t-1}}\right) \mid \mathbf{q}_{\mathbf{t}-\mathbf{1}}\right]=\mathbf{0}$. That is why the first moment does not change and

$$
E\left[X_{t}\right]=\boldsymbol{\pi}^{\prime} E\left[X_{t} \mid \mathbf{q}_{\mathbf{t}}\right]=\boldsymbol{\pi}^{\prime}\left(\begin{array}{l}
\mu_{1} \\
\mu_{2} \\
\mu_{3}
\end{array}\right),
$$

where $E\left[X_{t} \mid \mathbf{q}_{\mathbf{t}}\right]$ is the 3 -vector whose $i$ th element consists of $E\left[X_{t} \mid q_{t}=i\right]$.

In the next Proposition 3.2.1 we indicate a relation for the second central moment (variance) of the state-independent autoregressive first order Markov process.

Proposition 3.2.1. Let $\left\{X_{t}\right\}$ be the state-independent autoregressive first order Markov process

$$
X_{t}=\phi_{q_{t}}+\phi_{1} X_{t-1}+\varepsilon_{t},
$$

where $\phi_{1}$ is the autoregressive coefficient independent on the state (the same for all states $),\left|\phi_{1}\right|<1, \varepsilon_{t}$ is IID process $\approx N\left(0, \sigma_{\varepsilon}^{2}\right)$. Let $\boldsymbol{\pi}=\left(\pi_{1}, \pi_{2}, \pi_{3}\right)^{\prime}$ be the vector of the steady-state (unconditional) probabilities. Then for the second central moment of $\left\{X_{t}\right\}$ holds

$$
E\left[\left(X_{t}-\mu\right)^{2}\right]=\pi^{\prime}\left[\left(\begin{array}{c}
\mu_{1}-\mu \\
\mu_{2}-\mu \\
\mu_{3}-\mu
\end{array}\right) \otimes\left(\begin{array}{c}
\mu_{1}-\mu \\
\mu_{2}-\mu \\
\mu_{3}-\mu
\end{array}\right)+\left(\begin{array}{c}
\frac{\phi_{1}^{2} \sigma_{1}^{2}}{\left(1-\phi_{1}^{2}\right)} \\
\frac{\phi_{1}^{2} \sigma_{2}^{2}}{\left(1-\phi_{1}^{2}\right)} \\
\frac{\phi_{1}^{2} \sigma_{3}^{2}}{\left(1-\phi_{1}^{2}\right)}
\end{array}\right)+\left(\begin{array}{c}
\sigma_{\varepsilon}^{2} \\
\sigma_{\varepsilon}^{2} \\
\sigma_{\varepsilon}^{2}
\end{array}\right)\right],
$$

where $\mu$ is unpredictable mean value of $\left\{X_{t}\right\}, \sigma_{i}^{2}, i=1,2,3$ are the state-dependent variances, $\sigma_{\varepsilon}^{2}$ is the $(3 \times 1)$ vector of the variance of the IID process $\varepsilon_{t}$ and mark $\otimes$ is element by element multiplying operator of 2 vectors. 


\section{MOMENTS OF MARKOV-SWITCHING MODELS}

Proof. For the second central moment we get from the relation (6)

$$
\begin{aligned}
E\left[\left(X_{t}-\mu\right)^{2}\right]= & E\left[\left(\mu_{q_{t}}-\mu\right)^{2}+\phi_{1}^{2}\left(X_{t-1}-\mu_{q_{t-1}}\right)^{2}+\varepsilon_{t}^{2}\right] \\
& +2 \phi_{1} \operatorname{Cov}\left[\mu_{q_{t}}-\mu, X_{t-1}-\mu_{q_{t-1}}\right]+2 \operatorname{Cov}\left[\mu_{q_{t}}-\mu, \varepsilon_{t}\right] \\
& +2 \phi_{1} \operatorname{Cov}\left[X_{t-1}-\mu_{q_{t}}, \varepsilon_{t}\right] .
\end{aligned}
$$

The assumption that $\left\{\varepsilon_{t}\right\}$ is IID process $\approx N\left(0, \sigma_{\varepsilon}^{2}\right)$ and the independence between $\varepsilon_{t-1}$ and $q_{t}$ for $t=0, \pm 1, \pm 2, \ldots$ imply that all covariances above are equal to 0 and therefore

$$
E\left[\left(X_{t}-\mu\right)^{2}\right]=E\left[\left(\mu_{q_{t}}-\mu\right)^{2}+\phi_{1}^{2}\left(X_{t-1}-\mu_{q_{t-1}}\right)^{2}+\varepsilon_{t}^{2}\right] .
$$

Applying the steady-state probabilities and using the equations (6) and (7) we get

$$
\begin{aligned}
E\left[\left(X_{t}-\mu\right)^{2}\right] & =\boldsymbol{\pi}^{\prime} E\left[\left(X_{t}-\mu\right)^{2} \mid \mathbf{q}_{\mathbf{t}}\right] \\
& =\boldsymbol{\pi}^{\prime} E\left[\left(\mu_{q_{t}}-\mu\right)^{2} \mid \mathbf{q}_{\mathbf{t}}\right]+\boldsymbol{\pi}^{\prime} E\left[\phi_{1}^{2}\left(X_{t-1}-\mu_{q_{t-1}}\right)^{2} \mid \mathbf{q}_{\mathbf{t}}\right]+\boldsymbol{\pi}^{\prime} E\left[\varepsilon_{t}^{2} \mid \mathbf{q}_{\mathbf{t}}\right] \\
& =\boldsymbol{\pi}^{\prime}\left[\left(\begin{array}{c}
\mu_{1}-\mu \\
\mu_{2}-\mu \\
\mu_{3}-\mu
\end{array}\right) \otimes\left(\begin{array}{c}
\mu_{1}-\mu \\
\mu_{2}-\mu \\
\mu_{3}-\mu
\end{array}\right)\right]+\boldsymbol{\pi}^{\prime} \phi_{1}^{2}\left[\sum_{i=0}^{\infty} \phi_{1}^{2 i} \mathbf{P}^{\mathbf{i}}\left(\begin{array}{c}
\sigma_{1}^{2} \\
\sigma_{2}^{2} \\
\sigma_{3}^{2}
\end{array}\right)\right]+\boldsymbol{\pi}^{\prime}\left(\begin{array}{c}
\sigma_{\varepsilon}^{2} \\
\sigma_{\varepsilon}^{2} \\
\sigma_{\varepsilon}^{2}
\end{array}\right) \\
& =\boldsymbol{\pi}^{\prime}\left[\left(\begin{array}{c}
\mu_{1}-\mu \\
\mu_{2}-\mu \\
\mu_{3}-\mu
\end{array}\right) \otimes\left(\begin{array}{l}
\mu_{1}-\mu \\
\mu_{2}-\mu \\
\mu_{3}-\mu
\end{array}\right)\right]+\phi_{1}^{2}\left[\sum_{i=0}^{\infty} \phi_{1}^{2 i} \boldsymbol{\pi}^{\prime} \mathbf{P}^{\mathbf{i}}\left(\begin{array}{c}
\sigma_{1}^{2} \\
\sigma_{2}^{2} \\
\sigma_{3}^{2}
\end{array}\right)\right]+\boldsymbol{\pi}^{\prime}\left(\begin{array}{c}
\sigma_{\varepsilon}^{2} \\
\sigma_{\varepsilon}^{2} \\
\sigma_{\varepsilon}^{2}
\end{array}\right) \\
& =\boldsymbol{\pi}^{\prime}\left[\left(\begin{array}{c}
\mu_{1}-\mu \\
\mu_{2}-\mu \\
\mu_{3}-\mu
\end{array}\right) \otimes\left(\begin{array}{c}
\mu_{1}-\mu \\
\mu_{2}-\mu \\
\mu_{3}-\mu
\end{array}\right)\right]+\phi_{1}^{2}\left[\sum_{i=0}^{\infty} \phi_{1}^{2 i} \boldsymbol{\pi}^{\prime}\left(\begin{array}{c}
\sigma_{1}^{2} \\
\sigma_{2}^{2} \\
\sigma_{3}^{2}
\end{array}\right)\right]+\boldsymbol{\pi}^{\prime}\left(\begin{array}{c}
\sigma_{\varepsilon}^{2} \\
\sigma_{\varepsilon}^{2} \\
\sigma_{\varepsilon}^{2}
\end{array}\right) \\
& =\boldsymbol{\pi}^{\prime}\left[\left(\begin{array}{c}
\mu_{1}-\mu \\
\mu_{2}-\mu \\
\mu_{3}-\mu
\end{array}\right) \otimes\left(\begin{array}{c}
\mu_{1}-\mu \\
\mu_{2}-\mu \\
\mu_{3}-\mu
\end{array}\right)+\phi_{1}^{2}\left(1-\phi_{1}^{2}\right)-1\left(\begin{array}{c}
\sigma_{1}^{2} \\
\sigma_{2}^{2} \\
\sigma_{3}^{2}
\end{array}\right)+\left(\begin{array}{c}
\sigma_{\varepsilon}^{2} \\
\sigma_{\varepsilon}^{2} \\
\sigma_{\varepsilon}^{2}
\end{array}\right)\right] \\
& =\boldsymbol{\pi}^{\prime}\left[\left(\begin{array}{c}
\mu_{1}-\mu \\
\mu_{2}-\mu \\
\mu_{3}-\mu
\end{array}\right) \otimes\left(\begin{array}{c}
\mu_{1}-\mu \\
\mu_{2}-\mu \\
\mu_{3}-\mu
\end{array}\right)+\left(\begin{array}{c}
\frac{\phi_{1}^{2} \sigma_{1}^{2}}{\left(1-\phi_{1}^{2}\right)} \\
\frac{\phi_{1}^{2} \sigma_{2}^{2}}{\left(1-\phi_{1}^{2}\right)} \\
\frac{\phi_{1}^{2} \sigma_{3}^{2}}{\left(1-\phi_{1}^{2}\right)}
\end{array}\right)+\left(\begin{array}{c}
\sigma_{\varepsilon}^{2} \\
\sigma_{\varepsilon}^{2} \\
\sigma_{\varepsilon}^{2}
\end{array}\right)\right] .
\end{aligned}
$$

In the proof of the second central moment we used that $\sum_{i=0}^{\infty} \phi_{1}^{2 i}=\frac{1}{1-\phi_{1}^{2}}$ and $\boldsymbol{\pi}^{\prime}$ is the vector of the steady-state probabilities, where $\boldsymbol{\pi}^{\prime} \mathbf{P}^{i}=\boldsymbol{\pi}^{\prime}$.

As an illustration and for the comparison, using the system Wolfram Mathematica (version 8), we computed all central moments of the first order 


\section{ANNA PETRIČKOVÁ}

3-regime MSW model. For this purpose we used the hydrological time series, which are represented by the flow rates of 5 selected Slovak rivers:

- Hron (Banská Bystrica): Jan 1931-Dec 2008,

- Litava (Plastovce): Jan 1931-Dec 2008,

- Morava (Moravský Ján): Jan 1922-Dec 2008,

- Poprad (Chmelnica): Jan 1931-Dec 2004,

- Topla (Hanušovce): Jan 1931-Dec 2008.

The first 2 columns of Table 3.2 contain the second central moment sample estimates for the selected original and simulated MSW model data series. The third column displays variance computed with the formula derived in the Proposition 3.2 .1 .

TABLE 1. The 2nd central moment for original and simulated time series and calculated from derived formula (slanted). The state-independent case.

\begin{tabular}{|c|c|c|c|}
\hline Data $\backslash$ 2nd central moment & Observed data & Simulated data & From formula \\
\hline Hron & 3.4363 & 2.9625 & 2.7241 \\
\hline Litava & 2.5570 & 2.1126 & 2.2124 \\
\hline Morava & 0.7824 & 0.6741 & 0.6758 \\
\hline Poprad & 1.1739 & 1.0258 & 1.0413 \\
\hline Topla & 0.5373 & 0.4708 & 0.5219 \\
\hline
\end{tabular}

\subsection{State-dependent MSW models}

Let us have the stationary autoregressive first order Markov process with the state-dependent autoregressive coefficients

$$
X_{t}=\phi_{q_{t}}+\phi_{1, q_{t-1}} X_{t-1}+\varepsilon_{t}
$$

where we simplified notation $\phi_{0, q_{t}}=\phi_{q_{t}}, q_{t} \in\{1,2,3\}$.

For the state-dependent mean value holds

$$
\mu_{q_{t}}=\phi_{q_{t}}+\phi_{1, q_{t-1}} \mu_{q_{t-1}} .
$$

When we substitute $\phi_{q_{t}}$ in the basic model we get

$$
X_{t}=\mu_{q_{t}}+\phi_{1, q_{t-1}}\left(X_{t-1}-\mu_{q_{t-1}}\right)+\varepsilon_{t},
$$

where $\phi_{1, q_{t-1}}$ is the first-order autoregressive coefficient for the state $q_{t-1}$.

After substituting back and considering stationarity of the process

$$
\left(\left|\phi_{1, q_{t-1}}\right|<1\right)
$$

we obtain

$$
X_{t}-\mu_{q_{t}}=\sum_{i=1}^{\infty}\left(\prod_{j=1}^{i} \phi_{1, q_{t-1}}\right) \varepsilon_{t-i}+\varepsilon_{t} \text {. }
$$




\section{MOMENTS OF MARKOV-SWITCHING MODELS}

In the following we present the Proposition 3.3.1 the second central moment of the stationary autoregressive first order Markov process with the state-dependent autoregressive coefficients.

Proposition 3.3.1. Let $\left\{X_{t}\right\}$ be a stationary autoregressive first order Markov process with the state-dependent autoregressive coefficients

$$
X_{t}=\phi_{0, q_{t}}+\phi_{1, q_{t-1}} X_{t-1}+\varepsilon_{t},
$$

where $\phi_{1, q_{t-1}}$ are the autoregressive coefficients that depend on the state, $\left|\phi_{1, q_{t-1}}\right|<1, \varepsilon_{t} \approx \operatorname{IID} N\left(0, \sigma_{\varepsilon}^{2}\right)$. Let us have the vector of the stationary probabilities

$$
\boldsymbol{\pi}=\left(\pi_{1}, \pi_{2}, \pi_{3}\right)^{\prime} .
$$

Then for the second central moment of $\left\{X_{t}\right\}$ holds

$$
\begin{gathered}
E\left[\left(X_{t}-\mu\right)^{2}\right]=\boldsymbol{\pi}^{\prime}\left[\left(\begin{array}{c}
\mu_{1}-\mu \\
\mu_{2}-\mu \\
\mu_{3}-\mu
\end{array}\right) \otimes\left(\begin{array}{l}
\mu_{1}-\mu \\
\mu_{2}-\mu \\
\mu_{3}-\mu
\end{array}\right)\right]+ \\
\boldsymbol{\pi}^{\prime}\left[\left(\boldsymbol{\Phi}^{2}\left(\mathbf{I}_{3}-\mathbf{B} \boldsymbol{\Phi}^{2}\right)^{-1}\left(\begin{array}{c}
\sigma_{\varepsilon}^{2} \\
\sigma_{\varepsilon}^{2} \\
\sigma_{\varepsilon}^{2}
\end{array}\right)\right)+\left(\begin{array}{c}
\sigma_{\varepsilon}^{2} \\
\sigma_{\varepsilon}^{2} \\
\sigma_{\varepsilon}^{2}
\end{array}\right)\right],
\end{gathered}
$$

where $\mu$ is the unpredictable mean value of $\left\{X_{t}\right\}, \boldsymbol{\sigma}_{\varepsilon}^{2}$ is the $(3 \times 1)$ vector of the variance of the IID process $\varepsilon_{t}, \mathbf{I}_{\mathbf{3}}$ is the $(3 \times 3)$ identity matrix, $\mathbf{B}$ is the $(3 \times 3)$ matrix of transition probabilities for the "time-reversed" Markov chain that moves back in time, $\boldsymbol{\Phi}$ is the $(3 \times 3)$ diagonal matrix with the first order autoregressive coefficients of the regimes on the main diagonal and mark $\otimes$ is an element by element multiplying operator of 2 vectors.

P r o of. In the proof of the Proposition 3.3 .1 we use the proof of the Proposition 3.2.1. For the second central moment, considering the relation (10), it holds

$$
\begin{aligned}
E\left[\left(X_{t}-\mu\right)^{2}\right]= & E\left[\left(\mu_{q_{t}}-\mu\right)^{2}+\phi_{1, q_{t-1}}^{2}\left(X_{t-1}-\mu_{q_{t-1}}\right)^{2}+\varepsilon_{t}^{2}\right] \\
& +2 \operatorname{Cov}\left[\mu_{q_{t}}-\mu, \phi_{1, q_{t-1}}\left(X_{t-1}-\mu_{q_{t-1}}\right)\right]+2 \operatorname{Cov}\left[\mu_{q_{t}}-\mu, \varepsilon_{t}\right] \\
& +2 \operatorname{Cov}\left[\phi_{1, q_{t-1}}\left(X_{t-1}-\mu_{q_{t}}\right), \varepsilon_{t}\right] .
\end{aligned}
$$

The assumption that $\left\{\varepsilon_{t}\right\}$ is IID process $\approx N\left(0, \sigma_{\varepsilon}^{2}\right)$ and the independence between $\varepsilon_{t-1}$ and $q_{t}$ for $t=0, \pm 1, \pm 2, \ldots$ imply that all covariances above are equal 0 and therefore

$$
E\left[\left(X_{t}-\mu\right)^{2}\right]=E\left[\left(\mu_{q_{t}}-\mu\right)^{2}+\phi_{1, q_{t-1}}^{2}\left(X_{t-1}-\mu_{q_{t-1}}\right)^{2}+\varepsilon_{t}^{2}\right] .
$$




\section{ANNA PETRIČKOVÁ}

Then for the state-dependent second power we have

$$
\begin{aligned}
E\left[\left(X_{t}-\mu\right)^{2}\right]= & \boldsymbol{\pi}^{\prime} E\left[\left(X_{t}-\mu\right)^{2} \mid \mathbf{q}_{\mathbf{t}}\right] \\
= & \boldsymbol{\pi}^{\prime} E\left[\left(\mu_{q_{t}}-\mu\right)^{2} \mid \mathbf{q}_{\mathbf{t}}\right]+\boldsymbol{\pi}^{\prime} E\left[\phi_{1, q_{t-1}}^{2}\left(X_{t-1}-\mu_{q_{t-1}}\right)^{2} \mid \mathbf{q}_{\mathbf{t}}\right] \\
& +\boldsymbol{\pi}^{\prime} E\left[\varepsilon_{t}^{2} \mid \mathbf{q}_{\mathbf{t}}\right] .
\end{aligned}
$$

For the expression $E\left[\phi_{1, q_{t-1}}^{2}\left(X_{t-1}-\mu_{q_{t-1}}\right)^{2} \mid q_{t}=i\right]$ holds

$$
\begin{aligned}
& E\left[\phi_{1, q_{t-1}}^{2}\left(X_{t-1}-\mu_{q_{t-1}}\right)^{2} \mid q_{t}=i\right] \\
& =\sum_{j=1}^{3} E\left[\phi_{1, q_{t-1}}^{2}\left(X_{t-1}-\mu_{q_{t-1}}\right)^{2} \mid q_{t-1}=j \cap q_{t}=i\right] \cdot P\left(q_{t-1}=j \mid q_{t}=i\right) \\
& =\sum_{j=1}^{3} E\left[\phi_{1, q_{t-1}}^{2}\left(X_{t-1}-\mu_{q_{t-1}}\right)^{2} \mid q_{t-1}=j \cap q_{t}=i\right] \cdot b_{i j} .
\end{aligned}
$$

Label

$$
\boldsymbol{\sigma}_{\varepsilon}^{2}=\left(\begin{array}{c}
\sigma_{\varepsilon}^{2} \\
\sigma_{\varepsilon}^{2} \\
\sigma_{\varepsilon}^{2}
\end{array}\right),
$$

$\boldsymbol{\Phi}$ the $(3 \times 3)$ diagonal matrix of the autoregressive coefficients $\left(\phi_{1, r}\right)_{r=1,2,3}$ and B the $(3 \times 3)$ matrix of the transition probabilities $b_{i j}, i, j=1,2,3$ for the "time-reversed" Markov chain that moves back in time. Thus

$$
E\left[\phi_{1, q_{t-1}}^{2}\left(X_{t-1}-\mu_{q_{t-1}}\right)^{2} \mid \mathbf{q}_{\mathbf{t}}\right]=\mathbf{B} \cdot \boldsymbol{\Phi}^{2} E\left[\left(X_{t-1}-\mu_{q_{t-1}}\right)^{2} \mid \mathbf{q}_{\mathbf{t}-\mathbf{1}}\right] .
$$

From the relations (11) and (12) we get

$$
\begin{aligned}
& E\left[\left(X_{t}-\mu_{q_{t}}\right)^{2} \mid \mathbf{q}_{\mathbf{t}}\right]= E\left[\left(\phi_{1, q_{t-1}}\left(X_{t-1}-\mu_{q_{t-1}}\right)+\varepsilon_{t}\right)^{2} \mid \mathbf{q}_{\mathbf{t}}\right] \\
&= E\left[\phi_{1, q_{t-1}}^{2}\left(X_{t-1}-\mu_{q_{t-1}}\right)^{2} \mid \mathbf{q}_{\mathbf{t}}\right] \\
&+\overbrace{2 \operatorname{Cov}\left[\phi_{1, q_{t-1}}\left(X_{t-1}-\mu_{q_{t-1}}\right), \varepsilon_{t} \mid \mathbf{q}_{\mathbf{t}}\right]}^{=\mathbf{0}}+E\left[\varepsilon_{t}^{2} \mid \mathbf{q}_{\mathbf{t}}\right] \\
&= E\left[\phi_{1, q_{t-1}}^{2}\left(X_{t-1}-\mu_{q_{t-1}}\right)^{2} \mid \mathbf{q}_{\mathbf{t}}\right]+E\left[\varepsilon_{t}^{2} \mid \mathbf{q}_{\mathbf{t}}\right], \\
& \Rightarrow E\left[\left(X_{t}-\mu_{q_{t}}\right)^{2} \mid \mathbf{q}_{\mathbf{t}}\right]=\mathbf{B} \mathbf{\Phi}^{\mathbf{2}} E\left[\left(X_{t-1}-\mu_{q_{t-1}}\right)^{2} \mid \mathbf{q}_{\mathbf{t}-\mathbf{1}}\right]+\boldsymbol{\sigma}_{\varepsilon}^{2} .
\end{aligned}
$$


Because we assume the stationary process $\left\{X_{t}\right\}$, we can write:

$$
\begin{gathered}
E\left[\left(X_{t-1}-\mu_{q_{t-1}}\right)^{2} \mid \mathbf{q}_{\mathbf{t}-\mathbf{1}}\right]=\mathbf{B} \boldsymbol{\Phi}^{\mathbf{2}} E\left[\left(X_{t-1}-\mu_{q_{t-1}}\right)^{2} \mid \mathbf{q}_{\mathbf{t}-\mathbf{1}}\right]+\boldsymbol{\sigma}_{\varepsilon}^{\mathbf{2}}, \\
E\left[\left(X_{t-1}-\mu_{q_{t-1}}\right)^{2} \mid \mathbf{q}_{\mathbf{t}-\mathbf{1}}\right]-\mathbf{B} \boldsymbol{\Phi}^{\mathbf{2}} E\left[\left(X_{t-1}-\mu_{q_{t-1}}\right)^{2} \mid \mathbf{q}_{\mathbf{t}-\mathbf{1}}\right]=\boldsymbol{\sigma}_{\varepsilon}^{\mathbf{2}}, \\
\left(\mathbf{I}_{\mathbf{3}}-\mathbf{B} \boldsymbol{\Phi}^{\mathbf{2}}\right) E\left[\left(X_{t-1}-\mu_{q_{t-1}}\right)^{2} \mid \mathbf{q}_{\mathbf{t}-\mathbf{1}}\right]=\boldsymbol{\sigma}_{\varepsilon}^{\mathbf{2}}, \\
E\left[\left(X_{t-1}-\mu_{q_{t-1}}\right)^{2} \mid \mathbf{q}_{\mathbf{t}-\mathbf{1}}\right]=\left(\mathbf{I}_{\mathbf{3}}-\mathbf{B} \boldsymbol{\Phi}^{\mathbf{2}}\right)^{-1} \boldsymbol{\sigma}_{\varepsilon}^{\mathbf{2}}, \\
\Rightarrow(\text { from (12) }) \quad \\
E\left[\phi_{1, q_{t-1}}^{2}\left(X_{t-1}-\mu_{q_{t-1}}\right)^{2} \mid \mathbf{q}_{\mathbf{t}}\right]=\mathbf{B} \boldsymbol{\Phi}^{\mathbf{2}}\left(\mathbf{I}_{\mathbf{3}}-\mathbf{B} \boldsymbol{\Phi}^{\mathbf{2}}\right)^{-1} \boldsymbol{\sigma}_{\varepsilon}^{\mathbf{2},}
\end{gathered}
$$

and finally,

$$
\begin{aligned}
E\left[\left(X_{t}-\mu\right)^{2}\right]= & \boldsymbol{\pi}^{\prime} E\left[\left(X_{t}-\mu\right)^{2} \mid \mathbf{q}_{\mathbf{t}}\right] \\
= & \boldsymbol{\pi}^{\prime}\left[\left(\begin{array}{c}
\mu_{1}-\mu \\
\mu_{2}-\mu \\
\mu_{3}-\mu
\end{array}\right) \otimes\left(\begin{array}{l}
\mu_{1}-\mu \\
\mu_{2}-\mu \\
\mu_{3}-\mu
\end{array}\right)\right] \\
& +\boldsymbol{\pi}^{\prime}\left[\mathbf{B} \boldsymbol{\Phi}^{\mathbf{2}}\left(\mathbf{I}_{\mathbf{3}}-\mathbf{B} \boldsymbol{\Phi}^{\mathbf{2}}\right)^{-1}\left(\begin{array}{c}
\sigma_{\varepsilon}^{2} \\
\sigma_{\varepsilon}^{2} \\
\sigma_{\varepsilon}^{2}
\end{array}\right)\right]+\boldsymbol{\pi}^{\prime}\left(\begin{array}{c}
\sigma_{\varepsilon}^{2} \\
\sigma_{\varepsilon}^{2} \\
\sigma_{\varepsilon}^{2}
\end{array}\right) .
\end{aligned}
$$

Here we computed the second central moment of the first order 3-regimes state-dependent MSW model.

The first 2 columns of Table 3.3 contain the second central moment sample estimates for the selected original and simulated MSW model data series. The third column displays the variance computed with the formula derived in the Proposition 3.3.1.

For this purpose we also used the hydrologic time series like in the previous subsection.

TABLE 2. The 2nd central moment for selected original and simulated time series, and from formula. The state-dependent case.

\begin{tabular}{|c|c|c|c|}
\hline Data $\backslash$ 2nd central moment & Observed data & Simulated data & From formula \\
\hline Hron & 3.4363 & 2.4943 & 2.8742 \\
\hline Litava & 2.5570 & 1.9803 & 1.7497 \\
\hline Morava & 0.7824 & 0.6741 & 0.6549 \\
\hline Poprad & 1.1739 & 1.0149 & 1.0373 \\
\hline Topla & 0.5373 & 0.4561 & 0.5079 \\
\hline
\end{tabular}




\section{ANNA PETRIČKOVÁ}

\section{Conclusion}

In this paper we derived the relations for the second central moment of the state-independent and state-dependent MSW models - see Proposition 3.2.1 and 3.3.1.

In both cases we also computed the moments, using the system Wolfram Mathematica 8, and compared them with the variance calculated with the classical formula, defined in the system Mathematica.

From the Tables 3.2 and 3.3 we see that in calculating unconditional central moments of Markov switching models it is necessary to consider them as a function of the transition probabilities and parameters of the underlying state densities entering the switching process.

\section{REFERENCES}

[1] FRANSES, P. H.-VAN DIJK, F.: Non-Linear Time Series Models in Empirical Finance. Cambridge University Press, Cambridge, 2000.

[2] HAMILTON, J. D.: A new approach to the economic analysis of nonstationary time series subject to change in regime, Econometrica 57 (1989), 357-384.

[3] Hamilton, J. D.: Time Series Analysis. Princeton Univ. Press, Princeton, 1994.

[4] TIMMERMANN, J. D.: Moments of Markov switching models, J. Econometrics 96 (1999), 75-111.

[5] TONG, H.: Non-Linear Time Series: A Dynamical Systems Approach. Oxford University Press, Oxford, 1990. 